04

\title{
Пространственный фотоотклик, формфактор и требования к голографическим материалам
}

\author{
(C) С.А. Шойдин ${ }^{1}$, М.С. Ковалев ${ }^{2, \text { ॠ }}$ \\ ${ }^{1}$ Сибирский государственный университет геосистем и технологий, \\ 630108 Новосибирск, Россия \\ ${ }^{2}$ Московский государственный технический университет им. Н.Э. Баумана, \\ 105005 Москва, Россия \\ ฯ e-mail: m.s.kovalev@gmail.com
}

Поступила в редакцию 29.11.2019 г.

В окончательной редакции 20.03.2020 г.

Принята к публикации 28.03.2020 г.

Рассмотрены проблемы, возникающие при записи голограмм в объемных средах, проявляющиеся в первую очередь как пространственный резонанс поля записи голограммы с неоднородностями показателя преломления, обусловленными этой записью. А во-вторых, как перекрестное взаимодействие двух нелинейностей при записи голограмм - нелинейности дифракционной эффективности и нелинейности распределения по полю голограммы экспозиции и видимости регистрируемой голографической решеткой. Отмечается, что появление еще одной нелинейности - нелинейного фотоотклика голографического материала - при определенных условиях приводит не к снижению дифракционной эффективности, а к ее увеличению. В работе выделены основные классы объемных голографических сред - безрелаксационные динамические среды и среды с проявлением. Показаны ограничения максимально достижимой дифракционной эффективности для обоих типов сред. Сформулированы рекомендации по устранению нежелательных последствий обнаруженных эффектов, основанные на анализе основных закономерностей формирования фотоотклика с учетом пространственного резонанса и наличия формфактора.

Ключевые слова: голография, голографический материал, дифракционная эффективность, формфактор.

DOI: $10.21883 /$ OS.2020.07.49557.108-20

\section{Введение}

С самого начала работ по голографии $[1,2]$ перед разработчиками стоял принципиальный вопрос - на каком голографическом материале записывать голографическую информацию. История техники была очень успешной в связи с тем, что к моменту рождения голографии фотографическая индустрия развивалась уже довольно давно. Фотоматериалы на галогенсеребряных эмульсиях, разработанные для фотографирования и спектроскопии, уже выпускались на флагмане социалистической промышленности „ПО Славич“, сегодня АО „Компания Славич“. Это голографические материалы ПФГ-01, ПФГ-02, ПФГ-03 и ПФГ-04, известные во всем мире. Последний относится к бессеребряным материалам и изготавливается на основе бихромированного желатина. Такие материалы широко используются сегодня в голографии [3]. Однако мечта всех, кто занимается голографией, о 100\% преобразовании света в голографическое изображение на этих материалах не была достигнута. И не только изза технологических трудностей. Для тонких голограмм, используемых в схеме Э. Лейта и Ю. Упатниекса [2], известен теоретический предел, который не позволяет достичь дифракционной эффективности более $6.25 \%$ для амплитудных материалов, а для фазовых - 33.9\% [4,5]. Поэтому в течение многих лет внимание всех голографистов было сосредоточено на так называемых „толстых“ голографических слоях, в которых можно получить фазовые фотоиндуцированные изменения, т.е. изменения диэлектрической проницаемости вещества под действием света, приводящие к фотоиндуцированному изменению показателя преломления на длине волны восстановления [6].

Теоретически объемные фазовые голограммы, при отсутствии собственного поглощения материала, могут достигать 100\% дифракционной эффективности. Поэтому многие исследователи буквально бросились разрабатывать материалы, пригодные для записи таких голограмм, что привело к лавине публикаций, обзор которых можно увидеть, например, в [7]. Однако позже выяснилось, что большинство из них не идеальны. Они могут обладать сильным собственным поглощением, без которого невозможно обеспечить фоточувствительность, или иметь значительную анизотропию, искажающую дифракцию, как это бывает в кристаллах, или недостаточный диапазон фотоотклика, не позволяющий получить необходимый фазовый фотоотклик в материале, или его толщина недостаточна. Иногда в голограмме есть почти все, но она долго не держится. Одним словом, множество проблем не позволяют выбрать оптимальный материал или, по крайней мере, понять требования, которые нужно предъявлять к „идеальному“ голографическому материалу. Но это еще не все. 
В настоящей работе мы обращаем внимание на два физических эффекта, которые также не дают фазовым голографическим материалам достигать высокой дифракционной эффективности. Первый связан с эффектом, который можно назвать „пространственным резонансом поля записи голограммы с вызванными этой записью неоднородностями показателя преломления“, характерным для голограмм, записанных в динамических безрелаксационных средах, т.е. средах, формирующих фотоотклик непосредственно во время записи, без процесса проявления. Природа этого эффекта связана с взаимодействием частично созданной голографической решетки с еще поступающим (для продолжения записи) излучением от интерферирующих объектной и опорной волн, как это происходит в средах без проявления. Второй эффект существует и в средах с проявлением, он связан с наличием нелинейного распределения поля в пучках, которые записывают объемную (брэгговскую) голограмму. Этот эффект был обнаружен сравнительно недавно и получил название „формфактор“ голограмм.

\section{1. Запись голограмм в динамических безрелаксационных средах}

Среди динамических голографических материалов можно выделить те, фотоотклик которых определяется интегральной энергией света в течение всего процесса экспонирования, без стадии формирования скрытого изображения с последующим проявлением. Причем время релаксации индуцированного фотоотклика значительно превышает время его формирования. В таких средах самодифракция регистрирующих пучков происходит на дифракционных решетках, возникающих под их действием. Это обусловливает нестационарный энергетический обмен между регистрирующими пучками, особенности которого были исследованы на примере голографического материала Реоксан [8]. В настоящей работе проведен численный расчет дифракционной эффективности плоских пучков в динамических безрелаксационных средах с фотооткликом вида

$$
\varepsilon(r, t)=\varepsilon_{0}+\zeta=\varepsilon_{0}=\beta \int_{0}^{T}|E(r, t)|^{2} d t,
$$

где $\varepsilon_{0}-$ начальное значение диэлектрической проницаемости, $\beta$ - постоянная, определяющая чувствительность среды, $r=(x, y, z), E=a+b-$ суммарная амплитуда регистрируемых волн.

Динамика формирования голографической решетки, записанной в среде с таким откликом, для случая двух плоских волн с разными углами падения на поверхность среды описывается системой связанных волн

$$
\frac{\partial a}{\partial z}-\frac{i \pi}{2} R a-\frac{i \pi}{2} D b=0
$$

$$
\begin{gathered}
\rho \frac{\partial b}{\partial z}-\frac{i \pi}{2} R b-\frac{i \pi}{2} D a=0, \\
R(z, t)=\int_{0}^{T}\left[|a(z, t)|^{2}+|b(z, t)|^{2}\right] d t, \\
D(z, t)=\int_{0}^{T} a(z, t) * b(z, t) d t .
\end{gathered}
$$

Здесь система уравнений (2)-(4) аналогична [9], где $a(x, y), b(x, y)$ - объектная и опорная волны, а $R(z, t)$ и $D(z, t)$ - коэффициенты взаимодействия. Амплитуды $a, b$ нормированы к амплитуде $C$ на передней границе среды $z=0 ; z=z^{\prime} / L-$ координата, направленная вглубь среды толщиной $L$, перпендикулярной к поверхности; $\rho=\cos \theta_{b} / \cos \theta_{a}$, а $\theta_{a}, \theta_{b}$ - углы распространения волны в среде; $T=t / t_{0}$, где $t_{0}=\lambda \cos \theta_{a} / 4 \beta C 2 L$, $\lambda$ - длина волны. При таком выборе $t_{0}$ значение безразмерной переменной $t=1$ соответствует единичной дифракционной эффективности голограммы, записанной при $\rho=1$.

Для несимметричного падения пучков, когда $\rho \neq 1$, коэффициенты $R, D$ в (2) становятся функциями координаты $z$ и $t$, выражение для величины фотоиндуцированного изменения диэлектрической проницаемости $\xi(r, t)$ в соответствии с [9] имеет вид

$$
\xi(r, t)=\frac{\lambda \cos \theta_{a}}{2 L}\left[R+|D| \cos \left(K_{r}+\varphi\right)\right],
$$

где $\varphi(z, t)=\cos -1(\operatorname{Re} D /|D|), \quad K-$ фаза и вектор голографической решетки соответственно. Зависимость $R(z, t), D(z, t)$ была определена в результате численного решения системы (2) с граничными условиями $a(0, t)=b(0, t)=1$. По результатам расчета $R(z, t), D(z, t)$ определяются амплитуда $\xi \lambda|D| \cos \theta_{a} / L$ и фаза $\varphi$ решетки.

На рис. 1 приведены результаты численных расчетов зависимости амплитуды и фазы записанной голографической решетки, ее наклона в процессе записи и механизма самовоздействия регистрируемого излучения с формируемой им решеткой. Диаграммы фазы $\varphi(t)$ на рис. $1, a$ и нормированной амплитуды решетки показывают графики зависимости фазы $\varphi(t)$ и нормированной амплитуды решетки $\xi(t)=|D(1, t)|$ на обратной поверхности голограммы с параметром асимметрии регистрирующих пучков $\rho=0.1$. В отличие от случая симметричной записи пучками одинаковой интенсивности, величина $\xi$ растет нелинейно со временем, причем при $t>0.9$ амплитуда решетки начинает падать, т.е. за счет смещения интерферограммы (рис. $1, b, c)$ уже сформированная голографическая решетка разрушается. Нелинейность зависимости $\varphi(t)$ означает, что происходит не только наклон решетки, но и ее искривление (смещение от $A$ к $A^{\prime}$ на рис. $\left.1, c\right)$.

Голографическое проявление этих эффектов можно проследить, наблюдая поведение объектной волны $a$, 

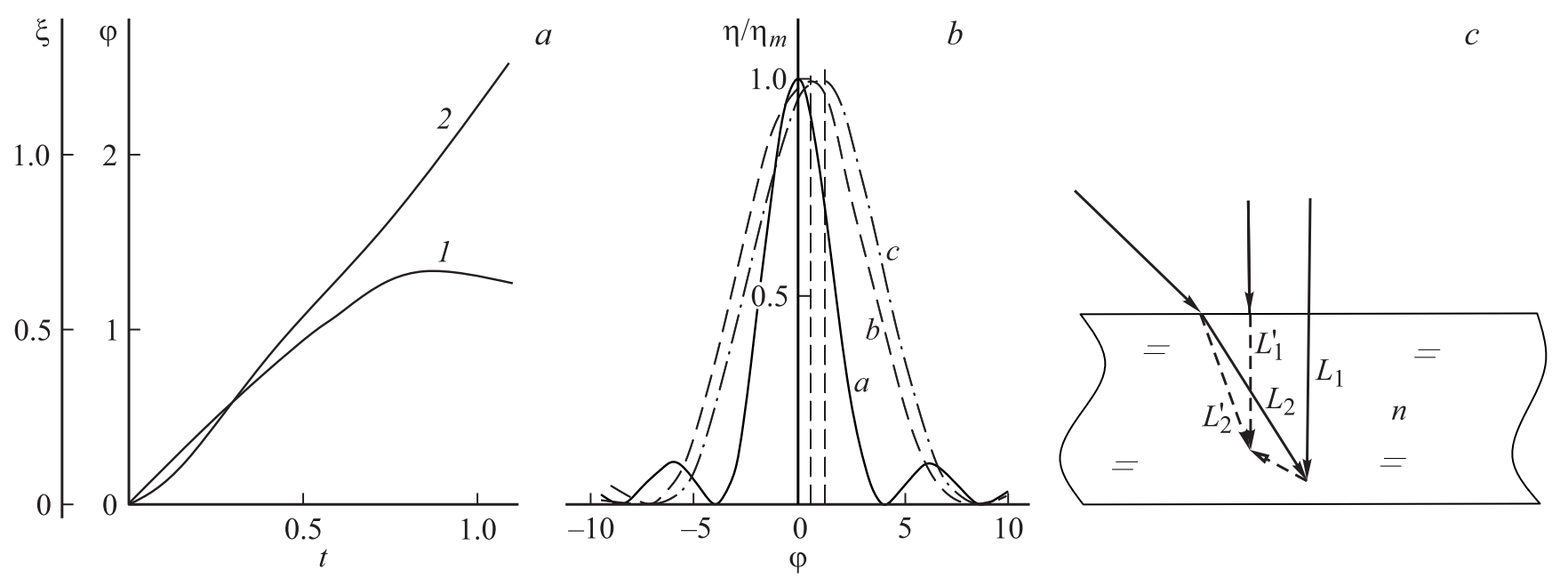

Рис. 1. Взаимодействие регистрирующего голограмму излучения с вызванным им фазовым фотооткликом голографического материала: $(a)$ нормированная амплитуда фотоиндуцированной диэлектрической постоянной $\xi(t)$ на задней поверхности $(z=L)$ голограммы (кривая 1$)$ и фаза $(\varphi)$ голографической решетки (кривая 2$) ;(b)$ нормированная дифракционная эффективность $\eta / \eta_{0}$ в зависимости от значения отклонения опорного пучка $\left(\varphi_{1}\right)$ при восстановлении. Сплошная кривая $(a)$ соответствует $t=1, \rho=1$; штриховые кривые $(b)$ случаям $t=0.5, \rho=0.5$ и $(c)-t=1, \rho=0.5$ соответственно; $(c)$ иллюстрация смещения точки из $A$ в $A^{\prime}$ для одинаковой разности эйконалов $L_{1}-L_{1}^{\prime}$, а также $L_{2}-L_{2}^{\prime}$ в процессе записи голограмм.

реконструированной на такой голографической решетке (5), т.е. решая задачу дифракции на такой решетке плоской волны в момент времени $t$. Предположим, что реконструирующая волна отличается от опорной волны при записи на небольшой угол $\delta$, лежащий в плоскости падения пучков при записи. В этом случае система уравнений для амплитуд волн при реконструкции отличается от (2) наличием членов $\gamma \sin \theta_{a}$ и $\gamma \sin \theta_{b}$ соответственно в левой части уравнений, где $\gamma=2 \pi \delta L / \lambda$. Угловая селективность голограммы определяется отношением интенсивности восстановленной волны $\eta=|a(z=1)| 2$ для различных $\delta$ к максимуму $\eta_{\max }$. Граничные условия для реконструкции записываются в виде: $a(0)=0, b(0)=1$. На рис. $1, b$ показаны графики угловой селективности при $\rho=0.5 ; \rho=1$ и $\cos \theta_{a}=1$ в разные моменты записи. Безразмерный параметр $\Gamma=2 \gamma \sin \theta_{b} / \pi$ характеризует угол отклонения $\delta$ реконструирующего пучка от опорного пучка при регистрации. Видно, что для $\rho \neq 1$ максимальная интенсивность реконструирующей волны достигается при отклонении реконструирующего пучка от опорного при записи. Величина сдвига кривой селективности при $t=1$ сопоставима с полушириной ее центрального лепестка. Сравнение кривых $(b)$ и $(c)$ показывает, что увеличение времени записи от $t=0.5$ до $t=1$ практически не приводит к уменьшению ширины кривой селективности.

Таким образом, асимметрия в геометрии записи голограмм плоскими пучками одинаковой интенсивности в динамической безрелаксационной среде приводит к существенным отличиям по сравнению со случаем симметричной записи, а также по сравнению с записью голограмм в проявляемой среде. Самодифракция регистрирующих волн на образуемой ими решетке приводит к изменению среднего наклона решетки в процессе записи, к искривлению решётки и к уменьшению амплитуды модуляции по глубине среды. Это проявляется в замедлении роста дифракционной эффективности и смещении брэгговского угла восстановления. В наиболее часто используемых случаях, когда $\rho>0.5$ и $t<1$, влияние кривизны решетки сравнительно невелико, а отмеченные различия в реконструкции Брэгга и снижении дифракционной эффективности обусловлены главным образом изменением наклона решетки, сопровождающимся уменьшением амплитуды ее модуляции по глубине голограммы.

\section{2. Резонанс спекл-поля}

Аналогичные эффекты экспериментально изучались при записи голографических решеток в голографических запоминающих устройствах (ГЗУ), когда в качестве предметного пучка формировалась спекл-структура при преобразовании Фурье матриц произвольно расположенных отверстий [10]. В ней экспериментально наблюдался пространственный резонанс спекл-поля с индуцированными им неоднородностями показателя преломления и было показано (рис. 2), что пик кривой селективности оказался смещенным.

Причиной такого смещения максимума дифракционной эффективности могут быть сразу два эффекта. Первый - это упомянутая ранее самодифракция при записи голограммы в динамической среде. Вторым является эффект пространственного резонанса спекл-волны, теоретически обнаруженный рядом авторов ранее [11]. Такой резонанс возникает в объеме среды при считыва- 

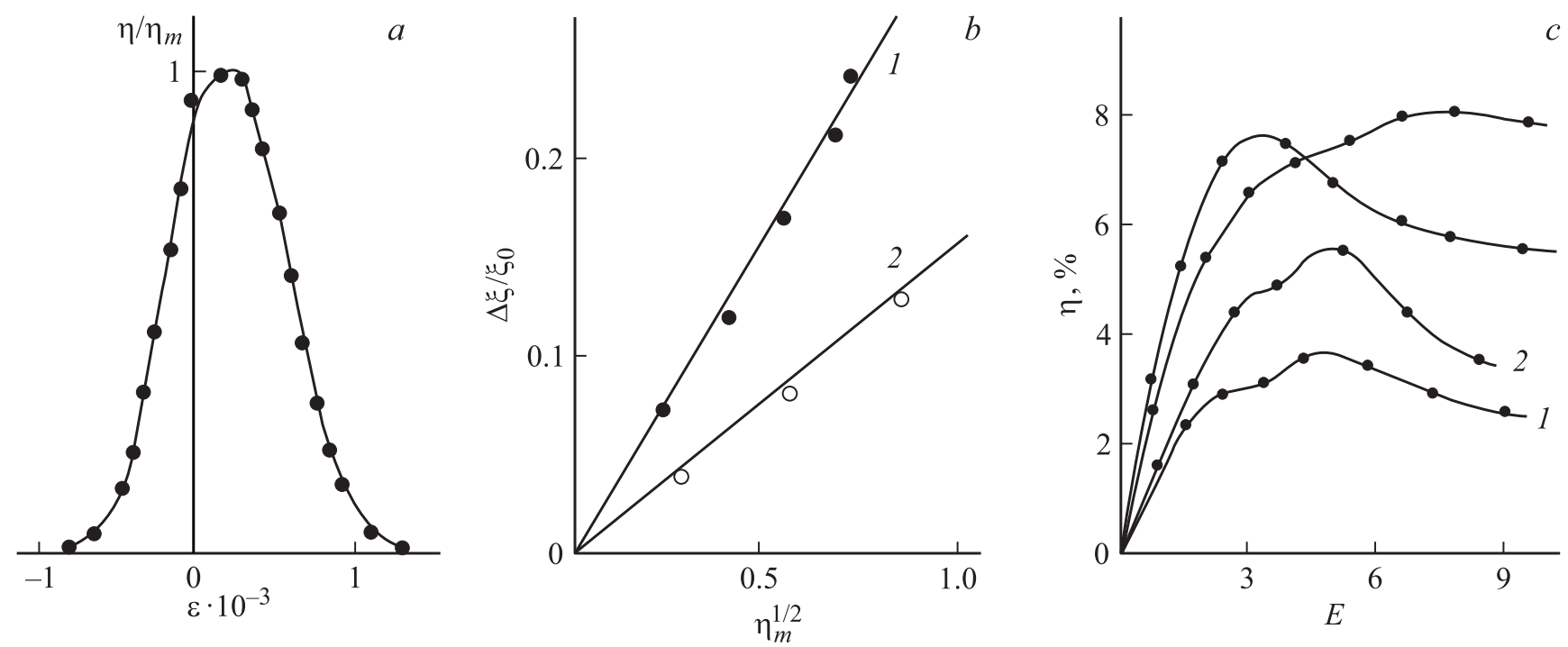

Рис. 2. Экспериментальная проверка самовоздействия записывающего голограмму излучения с формирующейся им голографической решеткой. (a) Экспериментально измеренные точки и аппроксимирующая их кривая нормированной величины дифракционной эффективности $\eta / \eta_{0}$ в зависимости от угла поворота $\xi$ плоской восстанавливающей волны относительно опорной при записи. Запись производилась НеNе-лазером на плоскую голографическую среду Реоксан толщиной $1 \mathrm{~mm}$. (b) Экспериментально измеренные точки сдвига оптимального угла восстановления $(\Delta \xi / \xi)$ (в единицах полуширины кривой селективности), при котором достигается максимальная дифракционная эффективность $\eta_{m} .1$ - для объектной спекл-волны, 2 - для объектной плоской волны $s$. (c) Экспериментально измеренные зависимости дифракционной эффективности от экспозиции при записи матриц голограмм в ГзУ: 1 - плоскость голограмм перед фокусом фурье-объектива, 2 - ближе к фокусу, 3 - в фокусе, 4 - за фокусом.

нии голограммы с неоднородностями спекл-структуры, записанной на голограмме.

Кроме того, наличие самодифракции на спекл-структуре записанной голограммы было экспериментально показано в работе [12]. На рис. 2, $а$ показаны кривые зависимости относительного сдвига оптимального угла $\Delta \varepsilon / \varepsilon$ реконструкции плоской волны от максимальной дифракционной эффективности $\eta_{m}$, которая нелинейно зависит от экспозиции, на примере материала Реоксан.

На рис. 2, $b$ показаны теоретические кривые 1 и 2 и соответствующие экспериментально измеренным значениям отклонения $\Delta \varepsilon / \varepsilon$ в зависимости от дифракционной эффективности, достигаемой на голограмме соответственно для регистрации объектного пучка, представляющего собой спекл-структуру (кривая 1) и плоский объектный пучок (кривая 2). Теоретически оценка такой кривой, полученная в [12], имеет вид $\Delta \varepsilon / \varepsilon=\left(\eta_{m}^{1 / 2} 2 \pi\right)$ $\left(<|A|^{2}><|B|^{2}>\right)^{1 / 2}$. Полученные экспериментальные данные хорошо ложатся на эту кривую.

Сравнение полученных результатов показывает, что при записи голограмм реальных объектов, представляющих собой сложную агломерацию плоских волн и, следовательно, имеющих спекл-структуру, смещение пика угловой селективности в динамических средах увеличивается в 2 раза за счет самодифракции восстановленного опорного пучка на регистрируемой спеклструктуре, что было названо в [13] пространственным резонансом. Точнее, данное взаимодействие интерференционной решетки с уже записанным фотооткликом мож- но назвать резонансно-диссипативным взаимодействием, так как они сначала взаимно усиливают друг друга, а затем разрушают.

Следует отметить, что указанная спекл-структура несколько меняется вблизи фокуса фурье.объектива и несколько по-разному ограничивает максимально достижимую дифракционную эффективность (рис. 2, b). При этом реальные максимумы на экспериментально полученных графиках всегда наступают при больших значениях экспозиции, чем при записи симметричных плоских пучков, соответствующих теории Когельника [5].

Лучшим способом борьбы с этими эффектами является создание проявляемых сред, в которых первичная энергия излучения создает скрытое изображение, как в галоидосеребряных средах, а уже другой физикохимический процесс преобразует это изображение в изменение параметров голографической среды, из которых, как следует из [5], фотоиндуцированное изменение показателя преломления является предпочтительным. Однако химический голографический проявитель в объемных средах трудно применять из-за проблем диффузии проявляющей среды внутри объемного голографического материала. Очевидно, физический процесс, например, сильное поле или воздействие температуры после записи голограммы, могло бы быть предпочтительным в данной ситуации.

Ограничение максимально достижимой дифракционной эффективности на рис. 2, с было связано не только с наклоном и кривизной интерференционных полос и 
пространственным резонансом спекл-структуры, но и с эффектом формфактора, обнаруженным позже.

\section{3. Формфактор и его влияние на запись голограмм}

Как было показано выше, динамической безрелаксационной среде имманентно присущи ограничения, которые на практике не позволяют достичь дифракционной эффективности, близкой к предельной [5]. Можно сделать вывод, что проявляемая среда, которая на первом этапе формирует только скрытое изображение, является идеальным выходом. Однако ниже мы покажем новый класс ограничений дифракционной эффективности и для таких голограмм, связанных с понятием формфактора.

Обычно при разработке новых голографических материалов и выборе удобных для решения технических решений большое внимание уделяется увеличению динамического диапазона фотоотклика среды [14-17]. Однако, как показано выше, использование большого динамического диапазона голографической среды для повышения дифракционной эффективности не всегда возможно.

Еще одна, чрезвычайно важная и даже, можно сказать, первичная причина ограничения дифракционной эффективности голограмм, которая существует даже в голограммах с механизмом записи скрытого изображения и с большими диапазонами фотоотклика, определяется влиянием так называемого формфактора голограммы. В работах [18-21] для разных случаев получены ограничения на максимально достижимую дифракционную эффективность $\eta_{m}$, а также получен коэффициент увеличения оптимальной экспозиции, необходимый для достижения ее максимума, называемый формфактором. Там же было отмечено, что формфактор сказывается на записи голограмм даже в проявляемых средах, но только при одновременном действии двух эффектов - нелинейности отклика, в данном случае $\eta_{m}(E, V)$, и неравномерности яркости в поле $E(x, y)$. При устранении одной из этих двух нелинейностей эффект формфактора вырождается, а величина достижимой дифракционной эффективности описывается классическим выражением, аналогичным полученному Когельником для плоской волны [5]. Степень приближения к этому классическому выражению определяется степенью приближения одной из двух вышеупомянутых нелинейностей к прямолинейному, равномерному распределению. Как показано в [1820], средняя дифракционная эффективность $\eta_{m}$ определяется по (6) и переходит к почти классической (7) (из [5]), когда произведение $\beta E(x, y) V(x, y)$ является постоянным в поле голограммы.

$$
\begin{gathered}
\eta_{m}=\left(\frac{1}{S}\right) \iint \sin ^{2}\{\beta(E) E(x, y) V(x, y)\} d x d y, \\
\eta=\Omega \sin ^{2}\{\Psi f(E V)\} .
\end{gathered}
$$

Здесь и ниже аналогично [18,19] экспозиция $E$ задается в нормированных единицах так, что $E=1$ изменяет фотоотклик голографического материала, приводя к увеличению параметра под синусом, равному одному радиану. Таким образом, экспозиция $E=\pi / 2$ при $\beta=1$ приводит к $\eta=100 \%$ при видности $V=1$ в соответствии с выражением, найденным Когельником, в которое вырождается (7) при $\Psi f(E V)=E V$.

На рис. 3 приведена зависимость $\eta_{m}(E, V)$, рассчитанная по (6) при записи гауссовыми пучками. На рис. 3, a показана двумерная зависимость от экспозиции и видимости, а на рис. $3, b$ приведены графики зависимости $\eta(E)$ от нормированной экспозиции $E$ классической дифракционной эффективности Когельника [5] (штриховая кривая) и $\eta_{m}(E)$, рассчитанные по формуле (6) для $V=1$. Видно, что $\eta_{m}(E)$ на рис. $3, a$ растет нелинейно, отставая от .(Е), как бы сначала взбираясь на первую ступеньку, а затем на следующую.

Это связано с тем, что, согласно (6), наиболее яркие участки $(x, y)$ голограммы гауссовых пучков достигают сначала максимальных локальных значений дифракционной эффективности, рис. 4, $a, b, c$, а затем уменьшают свой вклад в нее, когда значение аргумента в (7) перерастает $\pi / 2 ; \pi ; 3 \pi / 2$; и т. д. Следующий шаг увеличения $\eta_{m}(E, V)$ происходит при прохождении аргумента через $3 \pi / 4$ и т. д.

Отношение экспозиций, при которых дифракционная эффективность голограммы, записанной гауссовыми пучками, достигает первого максимума, к экспозиции, при которой дифракционная эффективность голограммы, записанной плоскими пучками, достигает своего первого максимума в [18-20], определяется как формфактор, т.е. коэффициент формы голограммы, определяемый формой пучков при ее записи и влияющий на скорость достижения максимально возможной дифракционной эффективности и величину этого максимума.

Категоричность указанных выше ограничений на максимально достижимую дифракционную эффективность смягчается при более пристальном взгляде на причины этого явления. Свободным параметром здесь является, например, видность $V$, точнее, комбинация трех параметров в (6), а именно экспозиции $E$, видности $V$ и чувствительности $\beta$, комбинируя которые по площади голограммы, можно добиваться максимальной похожести аргумента в (6) на константу по полю голограммы. В этом случае (7) вырождается в свой классический аналог из [5], что соответствует $\Psi=1$ в (7).

\section{1. Формфактор и негауссов пучок}

Указанные выше параметры $(E, V, \beta)$ имеют разную природу и часто могут быть искусственно изменены независимо друг от друга. Это помогает несколько оптимизировать влияние формфактора. Так, с использованием результатов работ $[21,22]$ были сделаны шаги к пониманию путей устранения этих ограничений. В [19] указывается, что при использовании негауссовых пучков 

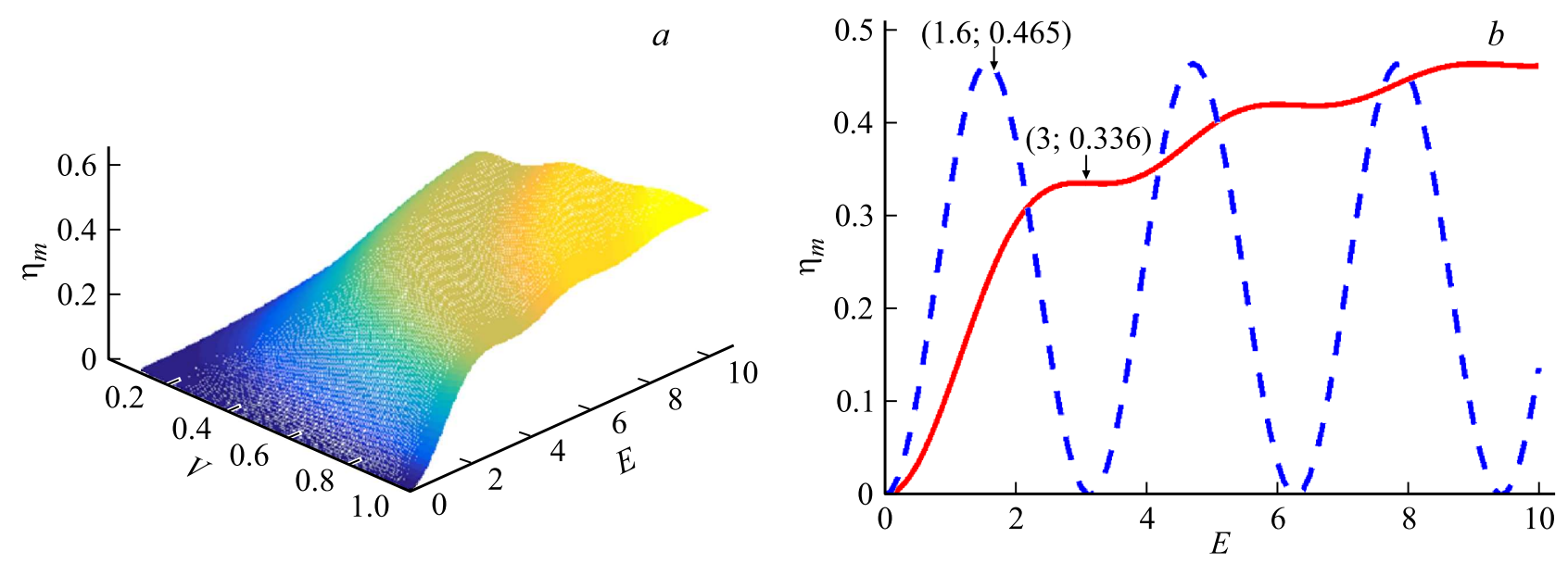

Рис. 3. Нелинейный рост средней дифракционной эффективности голограммы гауссовых пучков. (a) Зависимость $\eta_{m}$ от экспозиции $E$ и видности $V ;(b)$ - графики среза из рис. $1, a$ при $V=1-$ сплошная кривая и значение дифракционной эффективности по формуле Когельника для равномерного пучка (штриховая кривая), уменьшенная примерно в 2 раза по амплитуде до максимальных значений $\eta_{m}$ с целью удобства сравнения положений максимумов соответствующих кривых. Полученный формфактор $\Psi_{1}=1.6 / 3.0=0.53$.
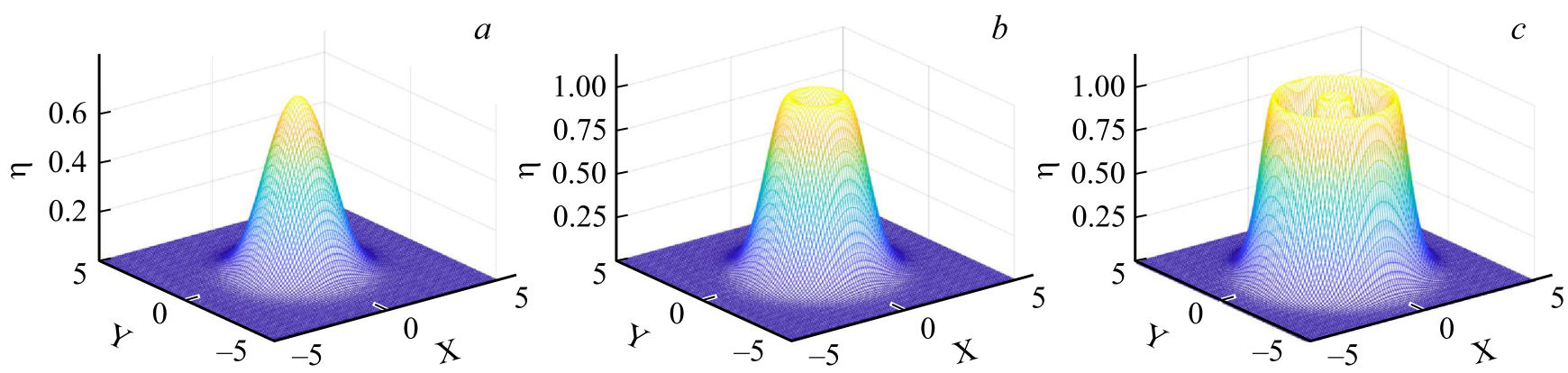

Рис. 4. Возрастающая от $(a)$ к $(c)$ экспозиция приводит сначала к росту локальной $\eta$, затем к провалу в центре и снова к росту.

(рис. 5) влияние формфактора ослабевает. Действительно, при использовании голограмм, записываемых лазерными пучками, описанными в $[21,22]$ (рис. 5, $a$ и $5, b)$, эффект формфактора может быть существенно уменьшен (рис. 5, $c$ и 5,d). Из графиков $\eta_{m}(E)$ на рис. $5, c, 5, d$, рассчитанных по (6), видно, что для таких пучков формфактор равен $\Psi_{2}=1.63 / 1.67=0.97$ и $\Psi_{3}=1.7 / 1.8=0.94$, т. е. онблизок к единице. При этом (7) приобретает классический вид из [5].

Аналогичным образом можно изменять по полю голограммы видность записывающих пучков $V(x, y)$ или светочувствительность голографического материала $\beta(x, y)$, тем самым уменьшая влияние формфактора.

\section{2. Формфактор и структура сложных изображений}

Несмотря на широкую применимость гауссовой модели пучка к записи голограмм, часто распределение яркости так называемых объектных пучков не соответствует форме гауссова поля в голограмме. Однако, как было отмечено в [23], большинство формально сложных пучков, имеющих многофакторное (хотя и не гауссово по полю) распределение яркости, в силу центральной предельной теоремы могут стремиться к гауссовой статистике распределения яркости (рис. 6). Это означает, что для голограмм, записанных такими пучками, можно также ожидать похожего проявления формфактора.

На рис. 6 показан портрет человека (рис. 6, $a$ ), гистограмма распределения яркости (рис. $6, b$ ) и рассчитанная (рис. $6, c)$ для такого изображения $\eta_{m}(E)$. Видно, что статистика распределения яркости в случае портретного изображения близка к гауссовой, а значение формфактора $\Psi_{4}=1.57 / 2.92=0.54$ практически совпадает с формфактором гауссовых пучков $\Psi_{1}=0.53$. Это означает, что условия записи голограмм таких сложных изображений будут аналогичны условиям записи гауссовых пучков.

При расфокусировке изображение изменяет статистику распределения яркости (рис. 7), а формфактор растет, но никогда не достигает единицы.

Таким образом, отклонения статистического распределения от гауссова, возникающие при расфокусировке (рис. 7), не приводят к существенному изменению формфактора. Допустимыми можно считать только такие 

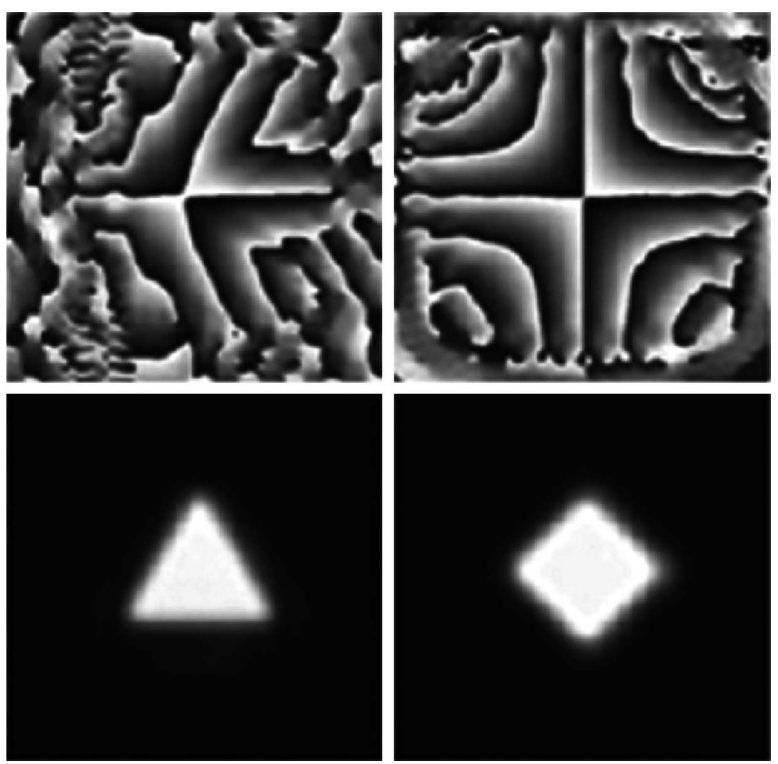

$c$

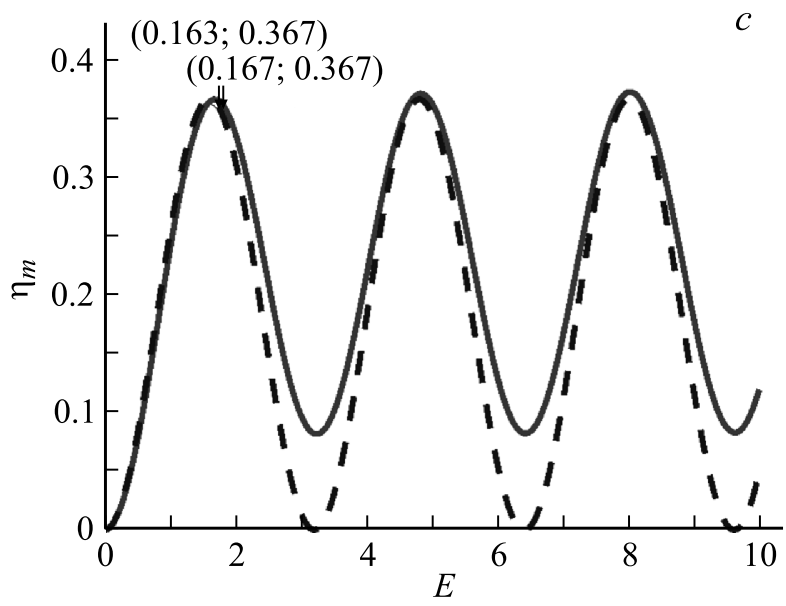

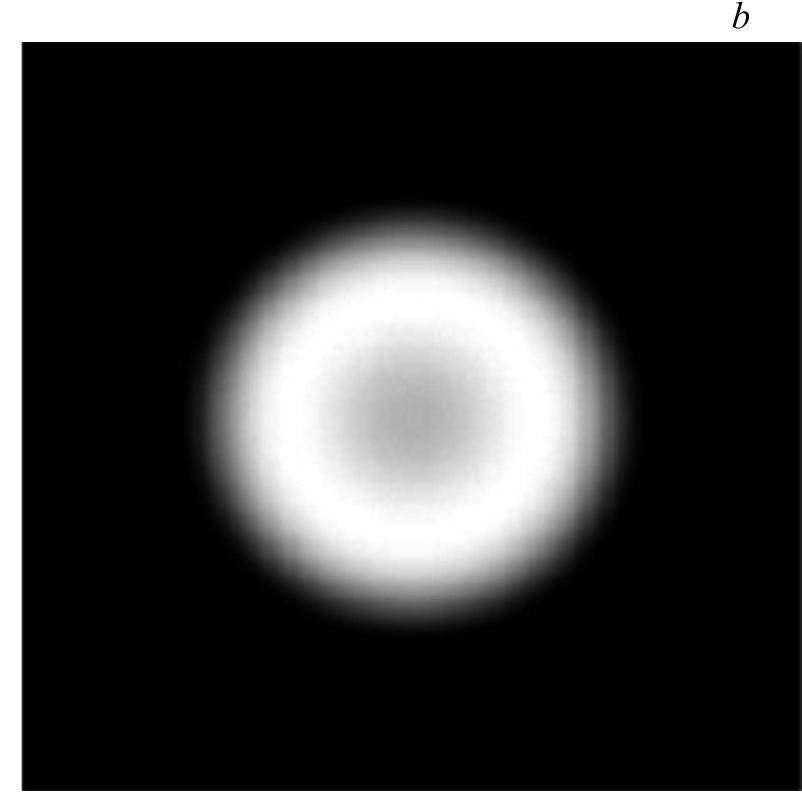

$d$

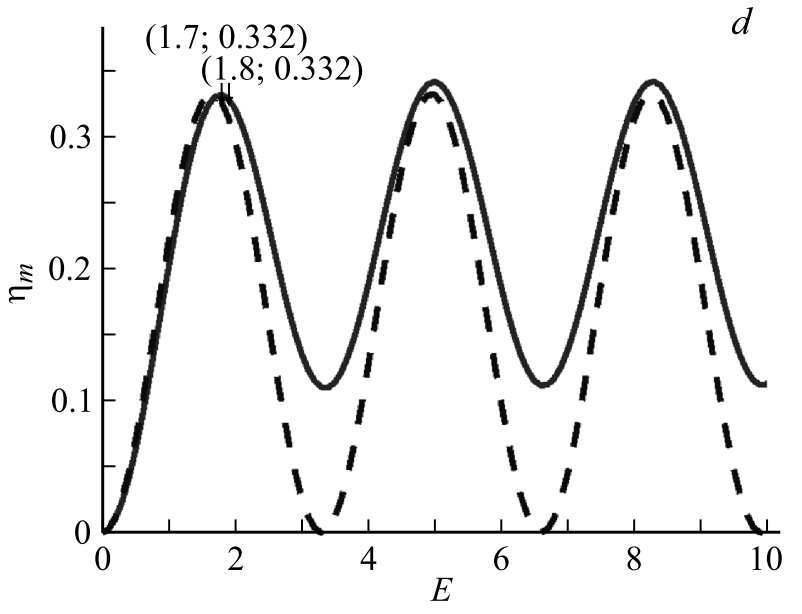

Рис. 5. Формфактор негауссовых пучков: (a) форма пучков на выходе лазера (снизу) при фазовой модуляции (сверху) из [23]; $(b)$ форма пучков на выходе лазера $[24] ;(c)$ средняя дифракционная эффективность $\eta_{m}(E)$, рассчитанная для случая $(a),(d)$ средняя дифракционная эффективность $\eta_{m}(E)$, вычисленная для случая $(b)$.

искажения изображения, которые не приводят к нарушению статистики сигнала (изображения), записанного на голограмме. Они могут включать в себя: расфокусировку, удаление объекта, изменение видности по полю и даже объема записанного изображения.

На рис. 7 и 8 соответственно показаны кривые $\eta_{m}(E)$, рассчитанные с помощью (6) для расфокусировки изображения (рис. 7) и при значительном удалении объекта от плоскости голограммы при записи (рис. 8) для голограммы того же портретного изображения.

Видно, что полученный формфактор также близок к формфактору для портрета и гауссова пучка. Это понятно, потому что при дальнейшей расфокусировке портретное изображение все больше приближается к пятну, напоминающему гауссов пучок. Однако преобразование Френеля, описывающее распространение пучка в свободном пространстве, изменяет статистику распределения яркости так, что значение формфактора практически не меняется. Это дает основание полагать, что формфактор подобен некоему инварианту движения, слабо зависящему от изменений записанного изображения.

\section{3. Формфактор и нелинейность фотографического отклика голографического материала}

Далее введем параметр нелинейности $\beta$, т.е. формирование фотоотклика будем принимать в виде (8), соответствующем экспериментально наблюдаемому в материале реоксан [8],

$$
\beta=\frac{a}{\left(1+1 /\left(a_{1} E\right)\right)} .
$$



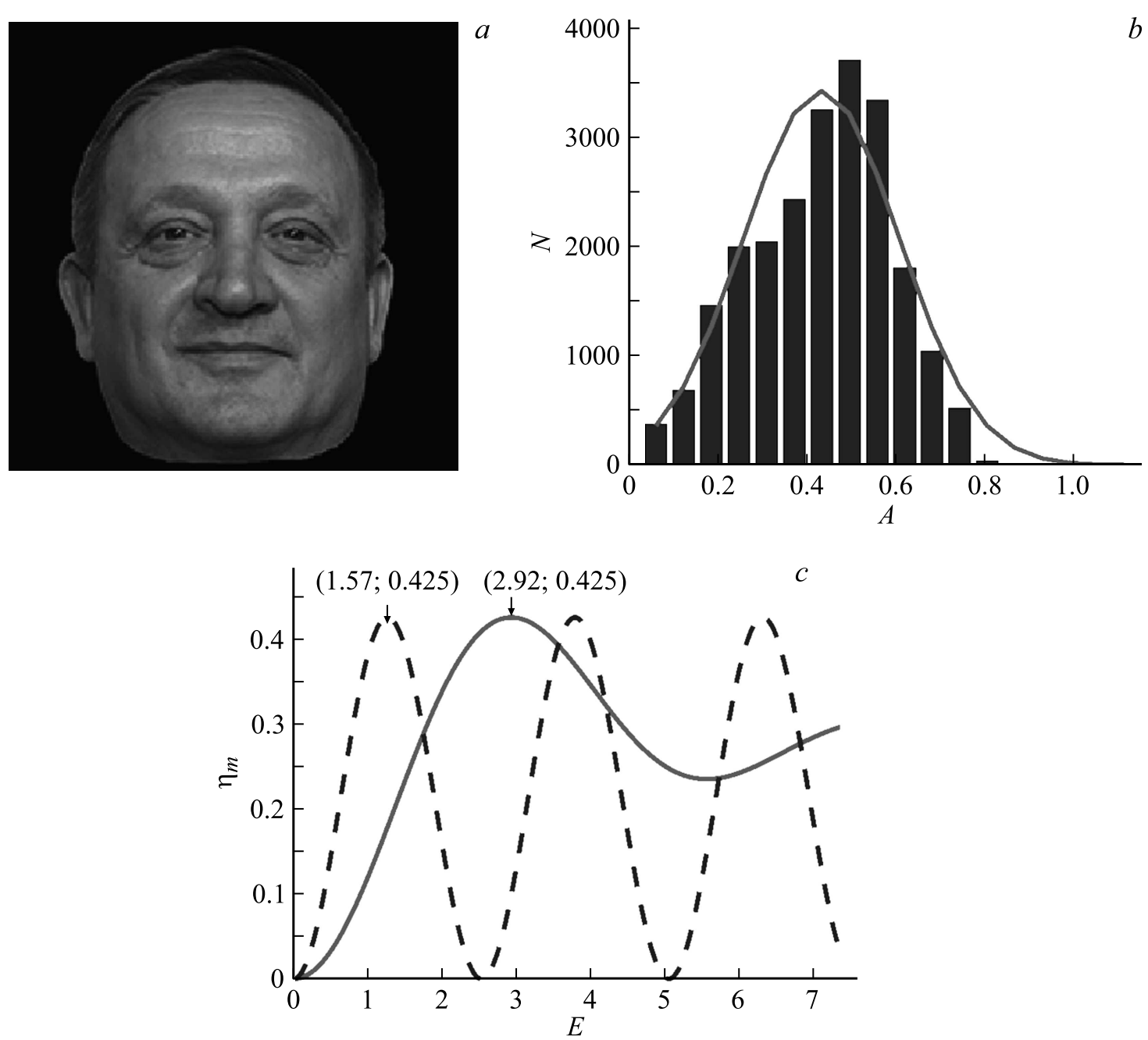

Pис. 6. (a) Портретное изображение; (b) гистограмма статистического распределения яркости портретного изображения, дисперсия отклонения от гауссова пучка $17.9 \%$; (c) дифракционная эффективность голограммы портретного изображения (сплошная кривая) и дифракционная эффективность равномерной по полю голограммы, описываемая теорией Когельника, уменьшенная по амплитуде для удобства сравнения (штриховая кривая). Полученный формфактор $\Psi_{4}=1.56 / 2.93=0.53$.

В соответствии с (6), (8) получены кривые дифракционной эффективности (рис. 9) и по ним рассчитаны значения формфактора [24], варьирующиеся соответственно от $\Psi_{7}=1.86 / 4.28=0.43$ до $\Psi_{8}=2.29 / 7.22=0.32$, и $\Psi_{9}=2.52 / 12.32=0.2$. При этом был замечен эффект увеличения максимальной дифракционной эффективности $\eta_{m}$ с увеличением нелинейности, причем достаточно заметный от $27 \%$ при $\alpha_{1}=0.1$ до $41 \%$ при $\alpha_{1}=0.3$. Это означает, что нелинейность отклика фотоматериала, используемого для записи голограммы, в случае неравномерной по полю яркости записывающих пучков приводит не к снижению дифракционной эффективности, а к ее увеличению.

Причиной этого является взаимодействие трех нелинейностей. Первая - это неравномерное, нелинейное распределение по полю интенсивности пучков, регистрирующих голограмму. Вторая - синусоидальная зависимость дифракционной эффективности фазовой голо- граммы от экспозиции. И третья - это нелинейный фотоотклик самого голографического материала. Наличие первой и второй нелинейностей создает эффект формфактора [18-20], а наличие третьей приводит к тому, что локальный вклад голографической дифракционной решетки в среднюю дифракционную эффективность, пройдя максимум, уменьшается медленнее, чем растут соседние области, все еще только стремящиеся к максимуму [24].

Следует отметить, что оба способа модификации изображений, записанных на голограмме (размытие и удаление объекта, рис. 7 и 8) при одинаковой нелинейности фотоотклика не дают существенного изменения $\eta_{m}(E)$, а также практически не влияют на величину оптимальной экспозиции, что еще раз подтверждает стабильный характер параметра, называемого формфактором. Таким образом, формфактор как для голограмм гауссовых пучков, так и для голограмм сложных, мно- 

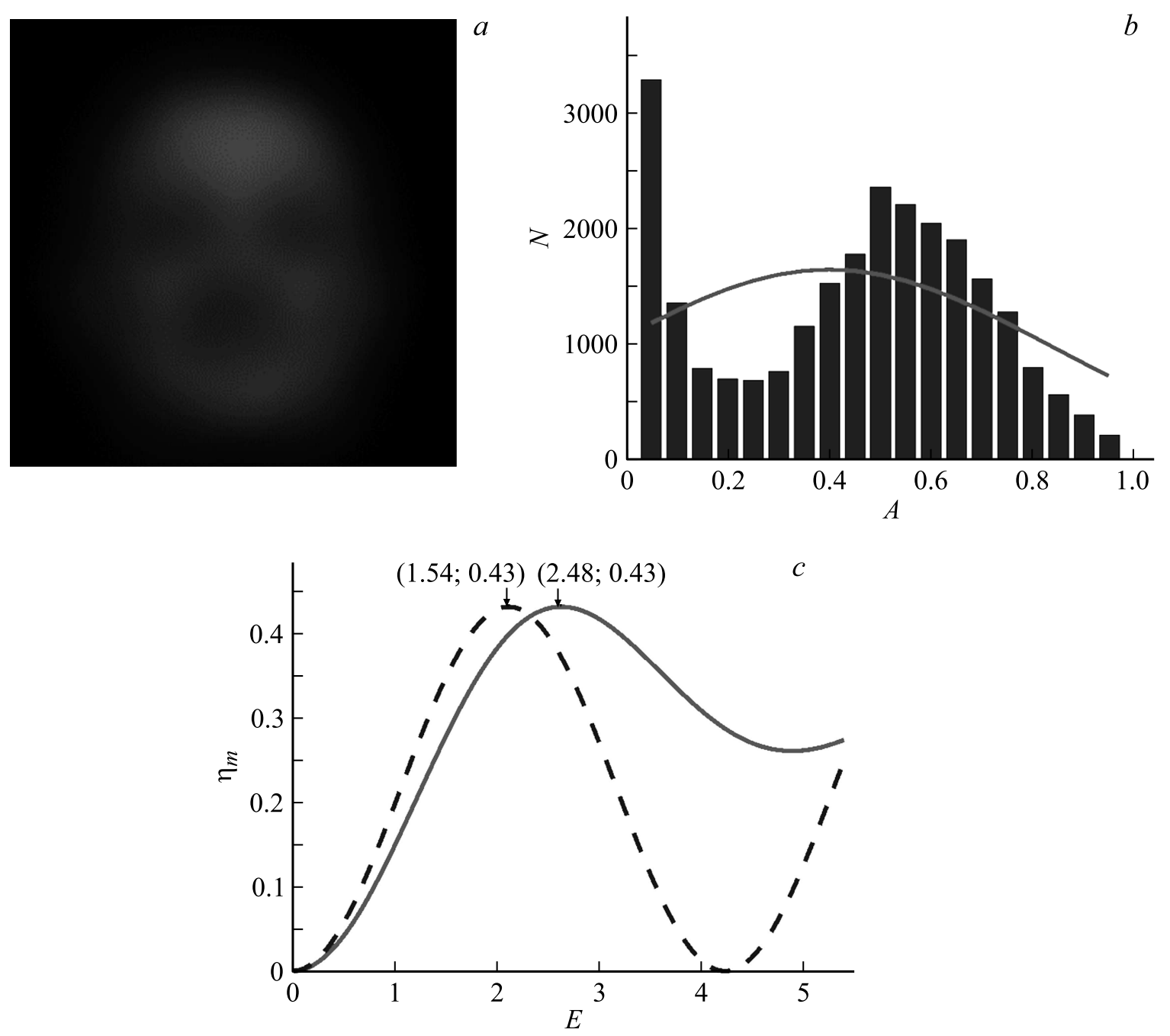

Pис. 7. (a) Портретное изображение, расфокусированное на $10 \%$; $(b)$ гистограмма статистического распределения яркости расфокусированного портретного изображения с дисперсией отклонения от гауссова пучка 45.2\%; $(c)$ дифракционная эффективность голограммы такого расфокусированного портретного изображения (сплошная кривая) и дифракционная эффективность равномерной по полю голограммы, рассчитанная по формуле Когельника, уменьшенная по амплитуде для удобства сравнения (штриховая кривая). Полученный формфактор $\Psi_{5}=1.54 / 2.48=0.62$.

гофакторных изображений подобен интегралу движения, так как он остается практически постоянным при значительных искажениях изображения, записанного на голограмме.

Само наличие формфактора существенно ограничивает достижимую дифракционную эффективность даже для голограмм, записанных на проявляемых материалах, где сначала формируется скрытое изображение, не влияющее на изменение фазы интерферирующих пучков в среде. Таким образом, название разд. 12 в [13] „Гимн объемным голограммам“можно считать несколько преждевременным. Следует сделать другой вывод: дальнейшее повышение дифракционной эффективности объемных голограмм связано с разработкой согласованных по параметрам голографических сред и лазеров для записи голограмм.

\section{Заключение}

При разработке новых голографических материалов приоритет следует отдавать проявленным объемно-фазовым материалам, чтобы избежать деструктивного влияния самодифракции и пространственного резонанса при записи. В то же время не следует увлекаться достижением рекордных значений динамического диапазона фотоиндуцированного отклика, поскольку сегодня одним из основных ингибирующих эффектов дифракционной эффективности отражательных голограмм является формфактор, который зависит одновременно от свойств голографической среды и от характеристик регистрирующего голограмму лазерного излучения. Также следует отметить, что нелинейная зависимость фотоотклика от экспозиции не всегда ограничивает максимально до- 

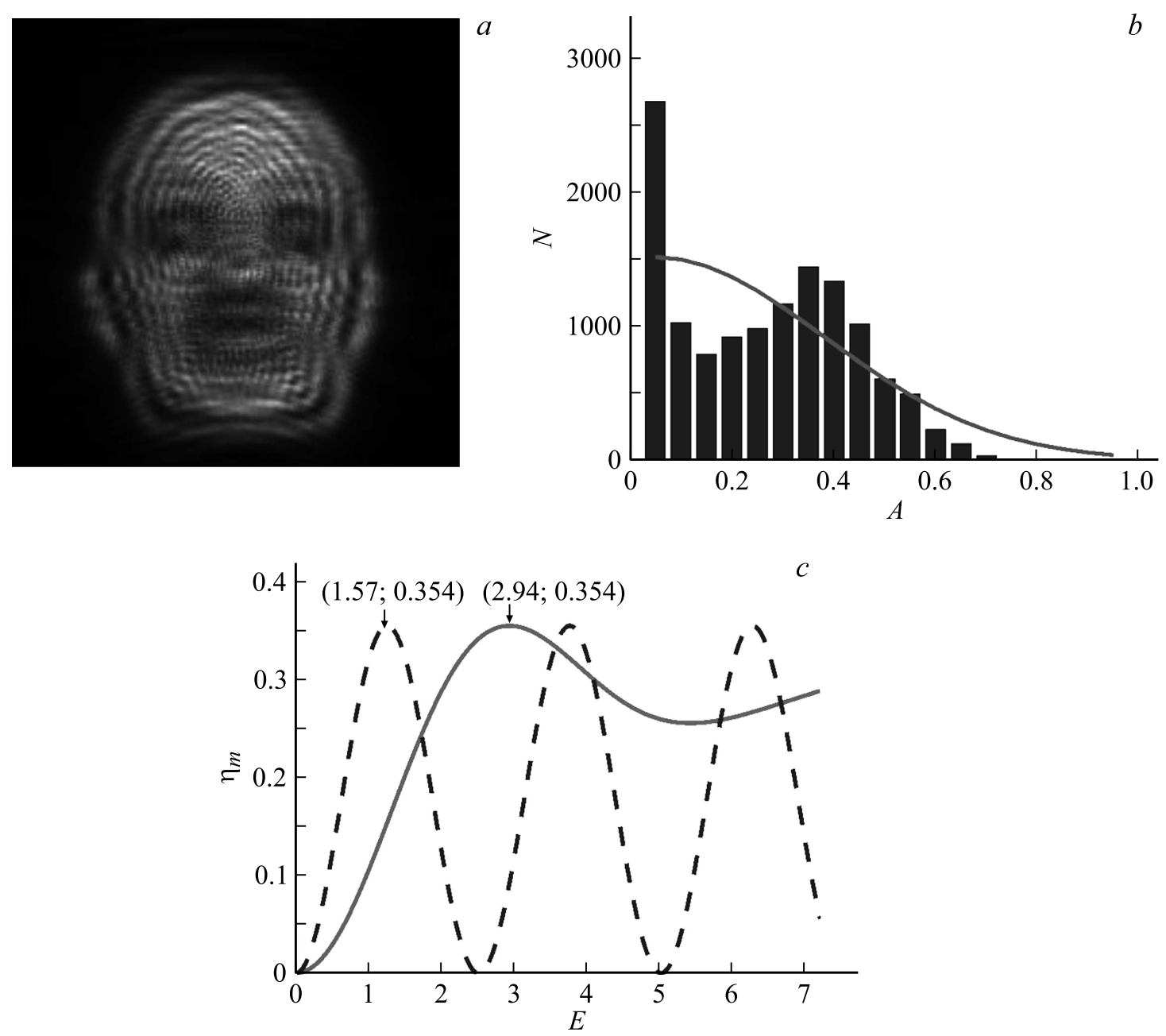

Рис. 8. Удаленное от плоскости голограммы изображение, представленное преобразованием Френеля: $(a)$ изображение в зоне дифракции; $(b)$ соответствующая гистограмма распределения яркости, дисперсия отклонения от гауссовой формы 47.9\%; (c) графики средней дифракционной эффективности по (1) при $V=1$ - сплошная кривая и штриховая кривая - значение дифракционной эффективности по формуле Когельника (2) при $\Psi_{f}(E V)=E V$ для равномерного пучка, полученный формфактор $\Psi_{6}=1.57 / 2.94=0.53$.

стижимую дифракционную эффективность, а наоборот, иногда за счет эффекта формфактора ее увеличивает.

Наиболее удачными, на наш взгляд, могут быть голографические материалы с химическим, термическим, электронным и др. проявлениями, когда первичный фотон только индуцирует процесс изменения показателя преломления (диэлектрической проницаемости), только создает канал, через который энергия химической реакции или физического поля многократно увеличивает воздействие на фотосреду (голографический материал), устраняя самопроизвольное взаимодействие волны и фотоматериала в процессе записи. Однако для объемной голографической среды необходима проницаемость ее объема для такого воздействия.

Главное, что следует из изучения свойств формфактора голограмм - это то, что некорректно рассматривать голографический материал отдельно, в отрыве от характеристик записывающего излучения. Разработка новых голографических систем должна координироваться с разработкой лазеров с измененным распределением интенсивности и видностью в поперечном сечении пучка, а также оптических схем (особенно некоторых их элементов), специально модифицированных для этой же цели. Таким образом, задача создания высокопроизводительных голографических дисплеев, систем памяти и других голографических проектов требует комплексных усилий разработчиков голографического материала, источников излучения и оптических схем для записи и восстановления.

\section{Послесловие}

Незадолго до окончания работы над этой статьей мы получили сообщение из Института прикладной физики Молдовы, Кишинев, о практическом интересе к формфактору и результатам, представленным в работах [18- 

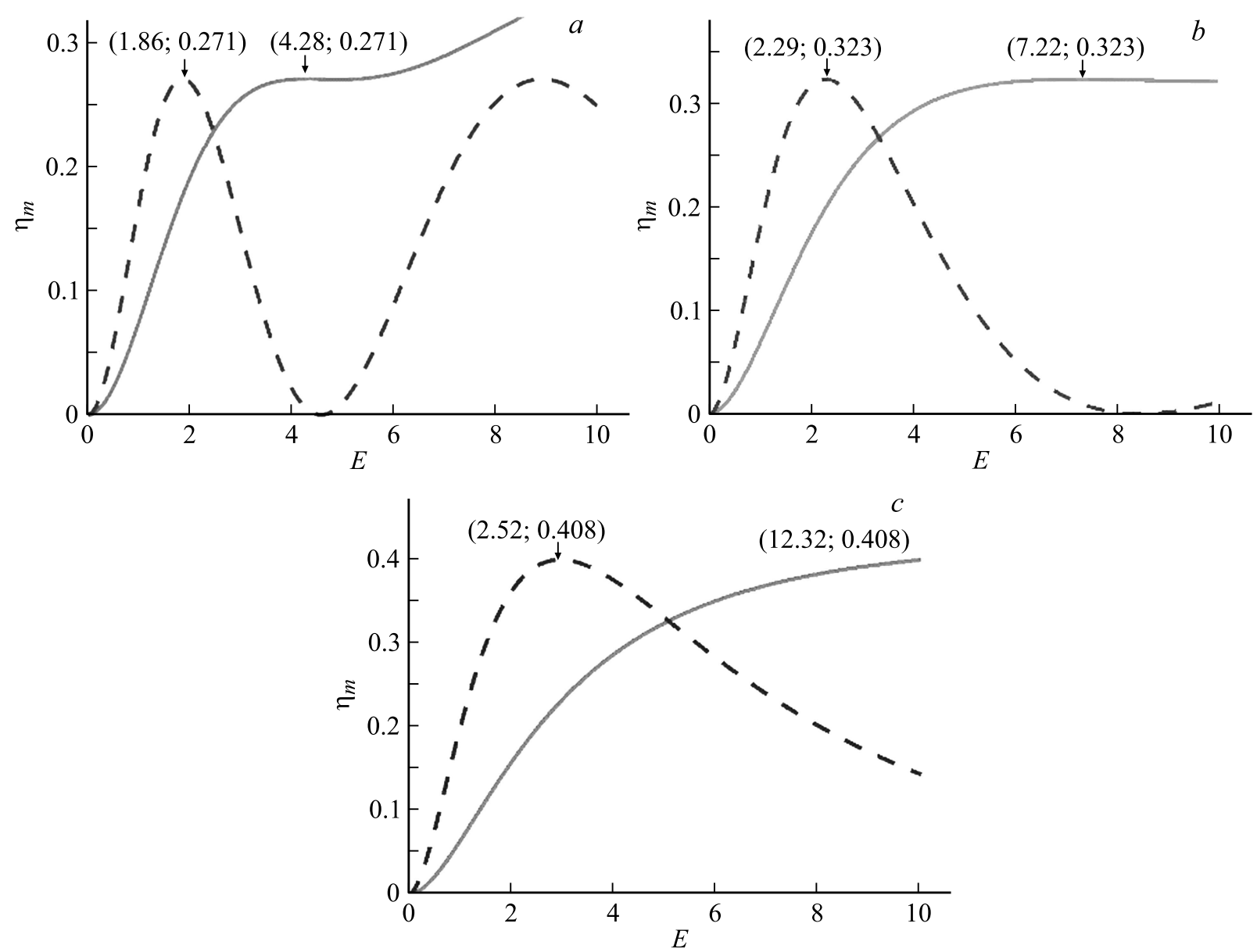

Рис. 9. Среднее значение дифракционной эффективности $\eta_{m}(E)$ голограмм гауссовых пучков для случаев нелинейности $\alpha=0.5$ и $\alpha_{1}=0.1(a) ; \alpha=0.5$ и $\alpha_{1}=0.2(b) ; \alpha=0.5$ и $\alpha_{1}=0.3(c)$.

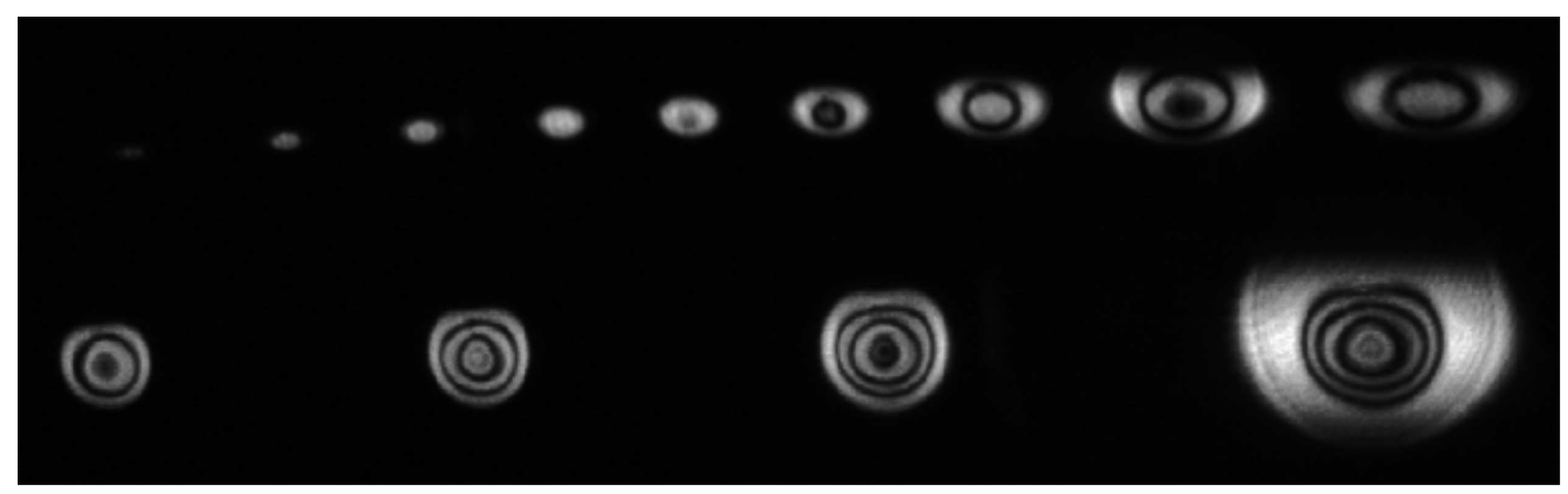

Рис. 10. Яркость восстановленного голограммой изображения с ростом ее экспозиции гауссовыми пучками.

20]. Даже были присланы результаты экспериментов по записи голограмм на халькогенидных стеклообразных полупроводниках и азополимерах (рис. 10).

Экспериментально наблюдаемое увеличение яркости $\eta_{m}(E)$ и колец ее провалов, аналогичное показанным на рис. 4, характеризующие наличие влияния формфактора при записи голограмм, говорит о более широком применении понятии формфактора не только для слу- чая дифракции Брэгга на объемных голографических решетках, но и для случая дифракции Рамана-Ната на поверхностных дифракционных решетах.

\section{Конфликт интересов}

Авторы заявляют, что у них нет конфликта интересов. 


\section{Список литературы}

[1] Денисюк Ю.Н. // Докл. АН СССР. 1962. Т. 144. № 6. C. 1275.

[2] Leith E.N., Upatnieks J. // J. Opt. Soc. Am. 1962. V. 52. P. 1123.

[3] Технические условия ПО „Славич“ на голографические материалы [Электронный ресурс]. Режим доступа: $\mathrm{http}: / /$ www.slavich.ru/?id=24

[4] Кольер Р., Беркхарт К., Лин Л. Оптическая голография: монография. М.: Мир, 1973. 698 с.

[5] Kogelnik H. // The Bell System Technical J. 1969. V. 48. N 9. P. 2909.

[6] Bobrinev V.I., Kovalev M.S., Odinokov S.B. // Russ. Phys. J. 2016. V. 58. P. 1457.

[7] Барачевский B.A. // Опт. и спектр. 2018. Т. 124. № 3. C. 371.

[8] Шойдин С.A., Сандер Е.A. // ОПт. и спектр. 1985. Т. 58. № 1. C. 200.

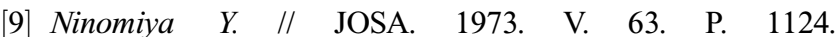
doi 10.1364/JOSA.63.001124

[10] Шойдин С.А., Шкунов В.В., Сандер Е.А. // ЖЭТФ. 1985. № 1. C. 116.

[11] Зельдович Б.Я., Пилипецкий Н.Ф., Шкунов В.В. // УФН. 1982. T. 138. № 2. C. 249.

[12] Shoydin S.A., Shkunov V.V., Sander E.A. // JETP. 1985. V. 61. N 1. P. 68.

[13] Зельдович Б.Я., Шкунов В.В., Яковлева Т.В. // УФН. 1986. T. 149. № 3. C. 511.

[14] Суханов В.И., Вениаминов А.В., Рыскин А.И., Никоноров Н.В. // Сб. трудов всероссийского семинара „Юрий Николаевич Денисюк - основоположник отечественной голографии“. СПб.: ФТИ им. А.Ф. Иоффе, СПбГУ ИТМО, НПК „ГОИ им. С.И. Вавилова“, 2007. С. 262.

[15] Шелковников В.В., Русских В.В., Васильев Е.В., Пен Е.Ф., Ковалевский В.И., Кучин И.А. // Оптич. журн. 2006. Т. 73. № 7. C. 65.

[16] Андреева О.В., Манухин Б.Г., Андреева Н.В. // Тез. докл. XIV международной конф. HOLOEXPO. 2017. С. 198.

[17] Шойдин C.A. // Тез. докл. XIV международной конф. HOLOEXPO. 2017. C. 198.

[18] Shoydin S.A. // Optical Memory and Neural Networks. 2016. V. 25. N 2. P. 95

[19] Шойдин C.A. // Компьютерная оптика. 2016. Т. 40. № 4. C. 501.

[20] Шойдин C.A. // Оптич. журн. 2016. Т. 83. № 5. С. 65.

[21] Волостников В.Г. Методы анализа и синтеза когерентных световых полей: монография. М.: Физматлит, 2014. 256 с.

[22] Чеботарев Г.Д., Латуш Е.Л., Пруиаков О.О., Фесенко А.А. // Квант. электрон. 2008. Т. 38. № 4. С. 299.

[23] Шойдин C.A., Трифанов А.В. // Компьютерная оптика. 2018. T. 42. № 3. C. 362. doi 10.18287/2412-6179-2018-423-362-368

[24] Шойдин C.A. // Автометрия. 2019. Т. 55. № 1. С. 35. 